Running head: GRADUAL DEVELOPMENT OF NATURE PREFERENCES

ACCEPTED ARTICLE (Journal of Environmental Psychology)

\title{
The gradual development of the preference for natural environments
}

Kimberly L. Meidenbauera*, Cecilia U. D. Stenfors ${ }^{a, b}$, Jaime Younga, Elliot A. Laydena, Kathryn

E. Schertz ${ }^{\mathrm{a}}$, Omid Kardanª, Jean Decetyc, \& Marc G. Bermana ${ }^{\text {* }}$

Affiliations:

a The Environmental Neuroscience Lab, Department of Psychology, University of Chicago

${ }^{\mathrm{b}}$ Aging Research Center, Department of Neurobiology, Care science \& Society, Karolinska

Institute, Solna, Sweden

c Child Neurosuite, Department of Psychology, University of Chicago

${ }^{*}$ Corresponding authors

Kim Lewis Meidenbauer

Email: kimlewis@uchicago.edu

Address:

5848 S University Avenue

Chicago, IL 60637 
GRADUAL DEVELOPMENT OF NATURE PREFERENCES

\begin{abstract}
Adults demonstrate aesthetic preferences for natural environments over urban ones. This preference has influenced theories like Biophilia to explain why nature is beneficial. While both adults and children show cognitive and affective benefits after nature exposure, it is unknown whether children demonstrate nature preferences. In the current study, 4-to-11year-old children and their parents rated their preferences for images of nature and urban scenes. Parents' preferences matched those of a normative adult sample. However, children demonstrated robust preferences for urban over natural environments, and those urban preferences significantly decreased with age. Nature exposure around the home and nature-related activities, as reported by parents, did not predict children's preferences. Children with more nearby nature, however, had lower reported inattentiveness, but interestingly, this was unrelated to children's preferences for nature. These results provide an important step into future research on the role of preference in how children and adults benefit from nature.
\end{abstract}

Key words: nature preferences, child development, nature exposure, Attention Restoration Theory, Biophilia, aesthetics, cognition 


\section{Introduction}

Humans have an incredibly strong preference for natural environments. People's preferences for natural environments over urban environments have been extensively documented (Kaplan \& Kaplan, 1989; Ibarra et al. 2017; van den Berg, Hartig, and Staats 2007). In fact, nature preferences are so strong that researchers have found that the distributions of adults' preference ratings for many kinds of nature and urban photos barely overlap (Kaplan, Kaplan, \& Wendt 1972; Berman et al., 2014; Kardan et al., 2015a). These preferences are also observed with real world dependent measures such as property values, as home buyers pay a premium for homes near nature (Crompton, 2001) and vacationers pay extra for rooms with natural sea views (Fleischer, 2012). These nature preferences are fairly universal as adults' preferences for nature are found cross culturally (Kaplan \& Yang, 1990; Ulrich, 1993). In fact, nature preferences are so strong that even within completely built structures, building interiors and exteriors that are perceived to be more natural are rated higher on aesthetic preference (Coburn et al., 2019).

Some theoretical accounts, such as Biophilia (Kellert \& Wilson, 1995) and Stress Reduction Theory (SRT) (Ulrich et al., 1991), suggest that these preferences for nature arise from humans evolving in natural environments. Though there is disagreement on how this might occur-whether this innate affinity is genetically programmed or works through a form of biologically-prepared learning-a common explanation for why nature is preferred is that only a tiny fraction of evolutionary history has occurred within our current urban environments, and the remainder in more natural environments.

In recent years, theories that suggest nature preferences arise from humans' evolutionary history have been called into question. Some critics of Biophilia and SRT 
emphasize the lack of strong empirical data to support such evolutionary accounts (Joye \& DeBlock, 2011; Joye \& van den Berg, 2011), and propose that positive feelings of restoration in nature are a byproduct of the ease with which humans are able to process features of the natural world (Joye, Steg, Unal \& Pals, 2015). This idea, referred to as the Perceptual Fluency Account (PFA), suggests that it is this lessened cognitive processing of stimuli in the natural world which underlies many of the observed benefits of nature (Joye \& van den Berg, 2011). While it can be argued why humans prefer nature, the persistent preferences for natural environments found in adults are well-established.

Though adults' preferences are well researched, very few studies have attempted to examine whether these preferences exist in children. One study conducted on this topic involved asking 9- to 12-year-old children to make a map or drawing of their favorite places. They found that the vast majority of the illustrations were outdoors, featuring lawns, playgrounds, and parks (Moore, 1986), suggesting that older children may display an affinity for nature that mirrors adult preferences. Those results, while highly informative, cannot speak to preferences for children under 9 years of age. In addition, from those results it is difficult to determine whether the children's preferences for natural spaces were indeed an indication of an affinity for nature, or a more general preference for areas associated with recreation. Other related research has addressed children's environmental reasoning, and found that elementary school children believe nature has intrinsic value and that preserving nature is important (Kahn, 1997). Children also seem to show visual preferences for certain types of natural environments (e.g., savannas and evergreen forests) over others (e.g., rainforests and deserts) (Balling \& Falk, 1982). Overall, while there is some preliminary evidence that children may appreciate and enjoy nature, 
no empirical work has directly examined natural versus urban preferences in children across a broad age range and across a varied stimulus set.

Knowing whether children do indeed prefer natural environments can inform two outstanding questions related to environmental preferences. First, examining children's preferences and their consistency (or lack thereof) during development can inform current debates about whether there is an innateness to nature preferences. This study may speak to whether nature preferences are genetically programmed and result from our evolutionary history (i.e. Kellert, 1993) or are learned through experience. Evidence for the latter hypothesis comes from recent research demonstrating that certain sounds and sound features are not preferred when the source of the sound is completely artificial (e.g., computer generated sounds) or obscured through scrambling, but become preferred when the sound is thought to be generated from a natural source, e.g., bird song (Van Hedger et al. 2019). Thus, there may not be anything intrinsically preferred about the features (visual, acoustic, tactile, etc.) of natural environments, and humans' preferences for nature may be heavily influenced by learned expectations.

Second, the present study can inform current theories about the role of nature preferences in the observed benefits of interacting with natural environments. In adults, these benefits include improvements in mood (Bratman, Hamilton, Hahn, Daily, \& Gross, 2015; Hartig, Evans, Jamner, Davis, \& Garling, 2003), positive physical health outcomes (Kardan et al., 2015b; Nielsen \& Hansen, 2007), and improved executive functioning (Berman, Jonides, \& Kaplan, 2008; Berman et al., 2012; Berto, 2005; Van Hedger et al., 2018; Schertz \& Berman, 2019). Though generally not as well studied, many of these same positive effects of nature are also found in children. Nature interventions have been shown 
to 1) decrease children's levels of stress, 2) reduce attention deficit hyperactivity disorder (ADHD) symptoms and 3) decrease levels of inattentiveness (Wells \& Evans 2003; Corraliza, Collado, \& Bethelmy, 2012; Faber Taylor \& Kuo, 2011; Amoly et al. 2014; Martensson et al. 2009).

Importantly, theories for how and why nature provides psychological benefits differ in the extent to which they believe nature preferences are required or relevant. Proponents of SRT argue that this preference for nature can explain some of the reduced stress and improved mood after interactions with natural environments, and this, in turn, can improve cognitive performance. Conversely, Perceptual Fluency theorists suggests that a positive response to and preference for nature is a consequence of the fluent processing of natural features rather than a cause of restoration (Joye \& van den Berg, 2011). Attention Restoration Theory (ART) (Kaplan, 1995; Kaplan \& Berman, 2010) does not require that natural environments be preferred to obtain the cognitive benefits. ART proposes that the cognitive improvements seen after nature interactions are not driven by mood or preference, but rather result from resting top-down directed-attention resources. Given that nature has been found salubrious for psychological functioning for both adults and children, if children do not prefer natural environments to the same extent as adults, this can inform extant theoretical accounts about the role of preference in obtaining cognitive benefits. Previous research on adults has suggested that preference and subsequent mood improvements may not be necessary to obtain the cognitive benefits (Berman et al., 2008; Stenfors et al., 2019), but results from this study would test whether preference is an important factor to obtain cognitive benefits in children. 
The primary goal of the current study was to examine whether this preference for natural environments exists in children, and whether children's preferences are consistent across development. To test whether children overall prefer natural or urban environments, we compared adults' and children's preferences for environments that varied on a spectrum of naturalness and preference. As adults' preference ratings for nature images are usually much higher than those for urban images, aesthetic preference and environment type are almost always confounded in studies using nature and urban stimuli (see Supplementary File 1). Therefore, the current study used nature and urban images that were equated on aesthetic preference from a separate study, to unconfound aesthetics (high vs. low preference) and environment type (nature vs. urban). This is critical to ensure that children's environmental preferences are not simply a result of a difference in general aesthetic preferences that have nothing to do with natural or urban environments per se. In other words, if preference and environment type are confounded, as they are in most studies because adults prefer nature, it would be impossible to know if any child preference differences were due to liking nature less or due to a non-specific difference in preference that has nothing to do with nature or urban environments. In our experiment, we can separate out these two possibilities.

If children do not show the same preference for nature as adults, this would suggest that there is not an innate, present-from-birth affinity for natural environments. This result would also imply that children's nature preferences may not be necessary to reap the cognitive benefits of interacting with the natural world. Similarly, if the preference for nature is due to learning, children's preferences for natural environments may develop over time. If children's preferences look very similar to those of adults', this would be a 
compelling case for a truly innate nature preference, consistent with a strong interpretation of the Biophilia Hypothesis (Kellert, 1993).

We also examined the developmental trajectory of environmental preferences to test the question of whether an affinity for nature is learned. If preferences change over time to look more similar to those of adults or increasingly relate to nature exposure with age, this would be evidence for a learned account. To directly test this question, analyses were conducted investigating children's preferences as a function of the naturalness of their home, school, and play environments, as well as parental preferences, and the interaction of these variables with age.

A final aim was to directly examine whether some of the observed cognitive benefits of nature related to nature preferences in the current sample. To test this, measures of emotional, social, and cognitive functioning (using the Strengths \& Difficulties Questionnaire) were collected and related to the amount of nature exposure and environmental preferences of the children. Overall, the current study sought to examine 1) whether children have environmental preferences that match those of adults, 2) whether these preferences are stable or change over time, 3) what factors may relate to individual differences and the development of environmental preferences, and 4) whether preferences relate to any observed cognitive/social/emotional benefits of nature in our sample. In addition to the fundamental importance of systematically testing nature preferences in a broad age-range of children and across a wide range of environments, this study will provide novel insights into current theories of nature preferences and the potential mechanisms of nature's psychological benefits. 


\section{Method}

\subsection{Participants}

\subsubsection{Sample Information}

Data from 251 children and 187 parents or guardians were collected. Twelve children were excluded from analysis due experimenter note that the child had clear difficulty understanding the task or failed to complete all trials of the task. The final sample included 239 children between the ages of 4 and 11 years and 182 adults (162 parents and 16 grandparents/other non-parent guardians). Gender split of the children in the final sample was relatively even (112 male, 127 female). Of the 239 usable children, 61 were siblings of another child participant. All study procedures were approved by the University of Chicago Institutional Review Board.

\subsubsection{Sample Size and Age Range}

The lower limit of the age range was selected based on a short pilot study previously conducted with 3- to 6-year-olds, which showed that children under 4 years of age had a very difficult time understanding the task. Our goal was to collect usable data from at least twenty children per one-year age bin, and we stopped data collection when we reached this goal. This number was determined from a quasi-power analysis using results from previous stimulus validation studies in our lab. These studies have found that obtaining preference ratings from about twenty adult participants is sufficient to gain reliable estimates of image preference (Kotabe, Kardan, \& Berman, 2017). Though the extent to which children would be similarly consistent in evaluating the images in this experiment were unknown, all planned analyses were to be conducted using the full sample of children 
from 4 to 11 years old, rather than analyzing within a certain year of age. As such, the problem of insufficient statistical power did not seem likely with a minimum of 20 children per year of age. Additional sample information by age bin can be found in Table 1.

Table 1. Child participant breakdown by age and gender

\begin{tabular}{lcr} 
Age Bin & N (\% of total) & \# F (\# M) \\
\hline 4 years & $21(8.8 \%)$ & $11(10)$ \\
\hline 5 years & $29(12.1 \%)$ & $16(13)$ \\
\hline 6 years & $42(17.6 \%)$ & $16(26)$ \\
\hline 7 years & $34(14.2 \%)$ & $15(19)$ \\
\hline 8 years & $30(12.6 \%)$ & $18(12)$ \\
\hline 9 years & $27(11.3 \%)$ & $15(12)$ \\
\hline 10 years & $30(12.6 \%)$ & $19(11)$ \\
\hline 11 years & $26(10.9 \%)$ & $17(9)$ \\
\hline Total & $\mathbf{2 3 9}$ & $\mathbf{1 2 7 ( 1 1 2 )}$
\end{tabular}




\subsubsection{Participant Geography and Nature Exposure}

As much of our recruitment occurred in a museum in Chicago which draws many tourists, the children in our study came from incredibly varied geographic locations. Of those who reported location either via zip code or city name ( $N=213), 42$ children came from Chicago, 52 from other parts of Illinois, 108 from other places in the US (across 23 other states), and 11 from other English-speaking countries (UK, Canada, Switzerland, Australia, and New Zealand).

Additionally, child participants came from locations that varied greatly on the extent to which they were urban or rural. Using National Land Cover Database data taken from US zip code, percentage of developed land was estimated for each zip code and used as a proxy for more versus less urbanized zip codes. A histogram of this data for all children who provided a zip code is found in Figure 1. The values for percentage developed range from $3 \%$ to $80 \%$, where zip codes with $3 \%$ developed land are primarily located in small, mostly

rural towns (populations of $<2,000$ people) and those with over $60 \%$ are primarily zip codes in major US cities. 
Figure 1. Level of urbanization for children's homes based on US zip code

Percentage of developed land is used as a proxy of urbanization, where lower values indicate less urbanized/developed zip codes and higher percentages indicate more urbanized/developed land. In blue are all children in the sample with US zip code information. In green, family-level data are presented which exclude redundancy of information due to possible siblings.

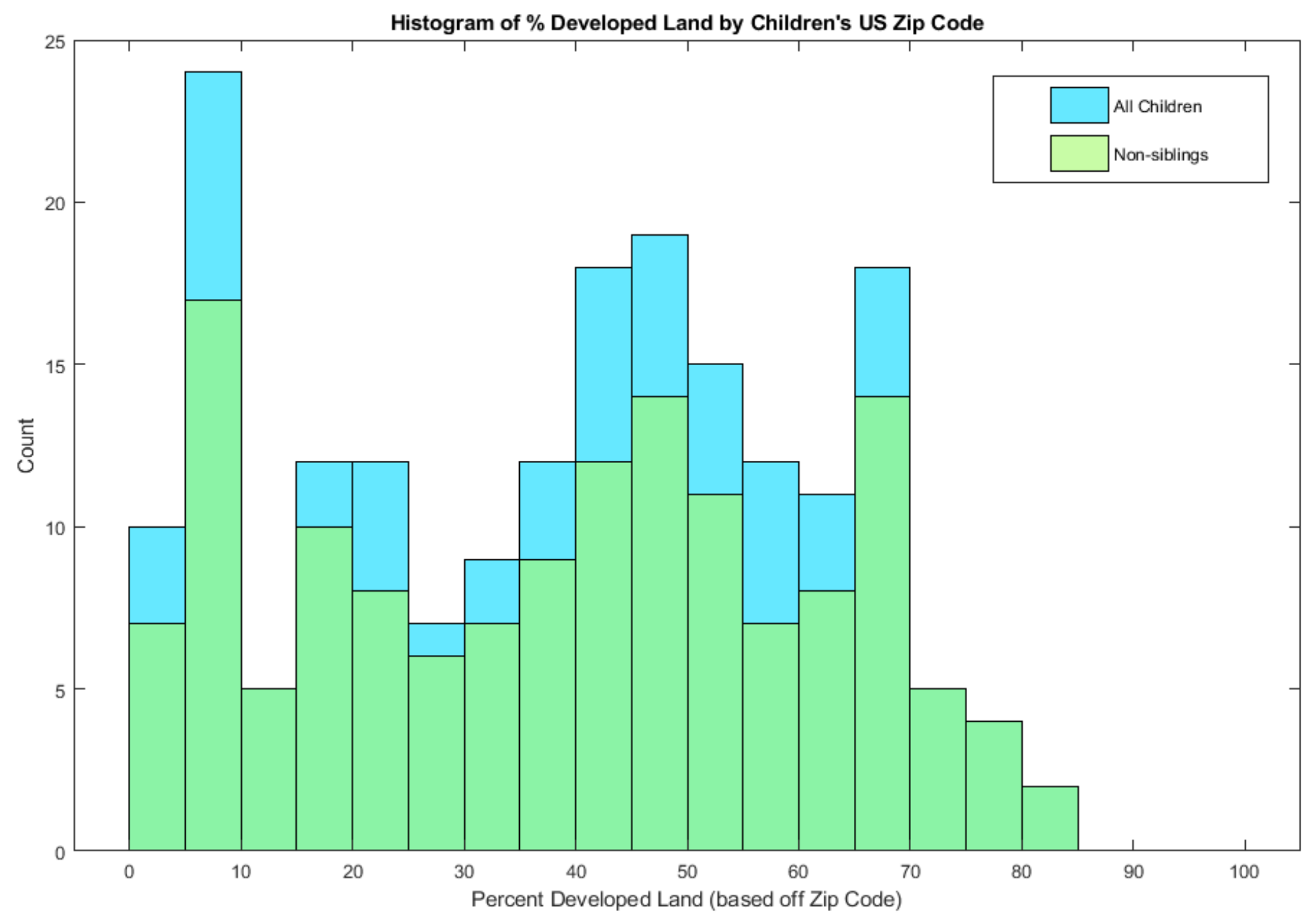




\subsubsection{Siblings in the Sample}

A number of children in our study were the sibling of another child in the study, leading to non-independence of these data points. Consequently, we also conducted analyses on a subset of our sample which did not include siblings. To create this subset, we pseudo-randomly selected one child out of all instances of siblings. We attempted to keep at least 22 children in each age bin by choosing more siblings in the outer age bins (which were typically sparser than the middle ages) and filling in other gaps as needed. Table 2 displays the breakdown of children by age included those analyses: i) without any siblings (all included), ii) in the sibling subset (1 child chosen for age balance), iii) the new noncorrelated sample (non-siblings + sibling subset), and iv) the original total sample (including siblings).

Table 2. Breakdown of all children and independent (non-correlated) subset

\begin{tabular}{|c|c|c|c|c|}
\hline Age & i) No siblings & $\begin{array}{l}\text { ii) Sibling } \\
\text { subset }\end{array}$ & $\begin{array}{l}\text { iii) Non-correlated } \\
\text { sample total }\end{array}$ & $\begin{array}{l}\text { iv) Original } \\
\text { sample }\end{array}$ \\
\hline $4 y$ & 13 & 7 & 20 & 21 \\
\hline $5 y$ & 20 & 2 & 22 & 29 \\
\hline $6 y$ & 24 & 0 & 24 & 42 \\
\hline $7 y$ & 15 & 7 & 22 & 34 \\
\hline $8 y$ & 11 & 11 & 22 & 30 \\
\hline $9 y$ & 11 & 11 & 22 & 27 \\
\hline $10 y$ & 13 & 9 & 22 & 30 \\
\hline $11 \mathrm{y}$ & 12 & 10 & 22 & 26 \\
\hline All & 119 & 57 & 176 & 239 \\
\hline
\end{tabular}




\subsection{Procedure}

\subsubsection{Instructions.}

Data collection occurred in lab and at a nearby museum. In the museum, experimenters directly approached families that appeared to have children in the correct age range to invite them to participate in a short research study. In both cases, parents (or guardians) provided informed consent for their child's participation before any additional study procedures occurred. Once parental consent and child assent were obtained, the child went through the picture sorting task procedure with an experimenter while another experimenter ran through the same task with the parent. The instructions for the task were as follows: "You are about to see sets of four pictures, and you will be asked to put them in order based on how much you like them. On the one end you will see a frowny face, and on the other you will see a smiley face. I want you to move these pictures around so that the pictures are in order of the one you like the least by the frowny face to the one you like the most by the smiley face. When you've put the photos in order of your least to your most favorite, you can press the green button to go onto the next set of pictures." All child participants had these basic instructions explained to them (and additional information and clarification added with 4- to 6-year old children), then completed four practice trials with the experimenter where they were asked to sort images of children's bedrooms before continuing onto the real task. To ensure that children understood the task, the researchers took the children through these practice trials very deliberately and carefully, asking children to verbally indicate their preference for the images as they moved them along the frowny-to-happy-face scale. Child participants who struggled with comprehending the task 
were still run through full procedures, but their lack of understanding was noted, and they were subsequently excluded from analysis. Adults were provided with the same general instructions but did not complete the practice trials.

\subsubsection{Stimuli Selection: Ratings from Validation Study.}

The specific stimuli used were taken from an image set which was rated on several attributes (including aesthetic preference and naturalness) in a previous validation study. In this validation study, adult participants rated a set of over 300 nature and urban images on a 1-7 Likert scale $(1=$ strongly dislike to $7=$ strongly like $)$. The preference ratings from this validation study were used to select the particular stimuli for the current study. Our goal was to find sets of nature and urban images which were rated very similarly on aesthetic preference to ensure that we'd be able to examine environmental preferences in children and any observed effects would not be attributable simply to differences in aesthetics. Full sized versions of all stimuli can be found here: https://osf.io/axn9q/?view_only=fa88e665f5a74885bd857d79f5a7ce4a, and a detailed explanation of the stimuli ratings, rationale for specific stimuli selection, and smaller versions of all images used can be found in Supplementary File 1.

\subsubsection{Stimulus Conditions}

There were two different image sets used in this experiment (parents and children always completed the task with the same image set). In each set, there were 6 categories of images based on previous normative adult evaluations of their aesthetic value from the validation study described above. These categories were high aesthetic value nature (two images), low aesthetic value nature (two images), high aesthetic value urban (two images), 
low aesthetic value urban (two images), very high aesthetic value nature (one image), and very low aesthetic value urban (one image). Images in the high aesthetic value nature and high aesthetic value urban categories were matched on preference, as were those in low aesthetic value nature and urban (see Table 3 for ratings of the current study stimuli from the validation study). The unmatched images (very high aesthetic value nature and very low aesthetic value urban) were included based on research in our lab that finds these images reliably elicit more extreme ratings in an adult sample. We have been unable to find sufficiently highly preferred urban images to match the very high aesthetic value nature (and nature to match the very low aesthetic value urban) which inevitably leads to a design in which conditions are not completely crossed. However, we chose to include these images as an additional, separate test of whether children's preferences map onto the preferences we see in adults. 


\section{Table 3. Average image aesthetic value pre-ratings}

Ratings on a 1-7 scale ( 1 = strongly dislike, 7 = strongly like) for images in each picture set. These ratings were gathered from a separate validation study with a normative adult sample. The images in each picture set were chosen with the goal of ensuring that the nature and urban images in the same aesthetic value category (i.e. High Aesthetic Value) were very closely matched on preference.

\begin{tabular}{rrrrrr}
\hline Very High & High & High & Low & Low & Very Low \\
Aesthetic & Aesthetic & Aesthetic & Aesthetic & Aesthetic & Aesthetic \\
Value & Value & Value & Value & Value & Value \\
Image & Image \#1 & Image \#2 & Image \#1 & Image \#2 & Image \\
\hline
\end{tabular}

\section{Picture Set 1}

\begin{tabular}{lllllll} 
Nature & 6.31 & 5.30 & 5.12 & 3.28 & 3.12 & \\
Urban & & 5.29 & 5.11 & 3.28 & 3.06 & 2.09 \\
\hline Picture Set 2 & & & & & & \\
Nature & 6.19 & 5.02 & 4.86 & 3.30 & 3.22 & \\
Urban & & 5.04 & 4.88 & 3.30 & 3.22 & 1.77 \\
\hline
\end{tabular}

\subsubsection{Task.}

The task was completed on a touch-screen tablet, which allowed participants to drag the images left and right to put them in the preferred order. Using this comparison approach rather than a Likert scale rating on single images was decided based on data from a pilot study. This pilot study showed that the use of Likert-type response scales on singular images for children in the lower end of our age range tended to result in a more binary decision (choosing the anchors of happy vs. frowny face) rather than along a continuum. The task included 10 trials where four images were shown at a time. The presentation of images was randomized across trials and across starting positions within a trial. Because of this randomization, any set of four images from the 10 image set could 
appear in a trial, but the task used an algorithm to ensure that each of the 10 images were compared to every other image in the set at least once (see Figure 2 for a display of the task).

\section{Figure 2. Task Design}

Upper panel depicts a trial from the practice rounds. Lower panel depicts a sample trial from the actual experiment, which contains images in the following conditions (from left to right): high aesthetic value nature, high aesthetic value urban, low aesthetic value urban, low aesthetic value nature.
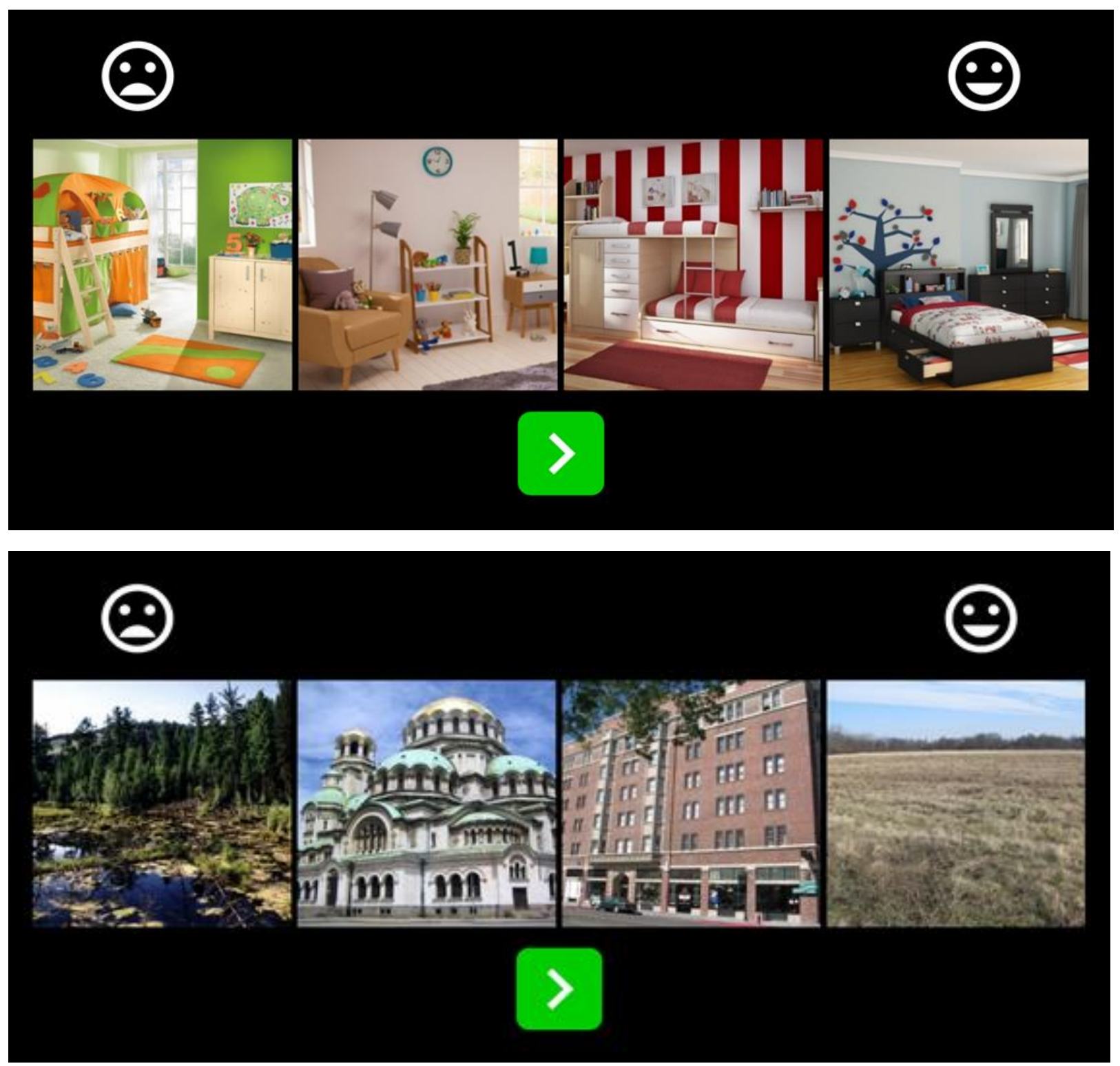


\subsubsection{Additional Measures.}

Parents were also asked to complete a number of optional questionnaires about their child. Standard demographic measures were collected (birthdate, gender, ethnicity, household income, parental education) as well as zip code, which was used to calculate objective greenspace/land cover types from the 2011 National Land Cover Database (NLCD). From the NLCD data, we calculated amount of natural features nearby (summed coverage of values for water, deciduous forest, evergreen forest, mixed forest, shrub, grassland, pasture, cultivated land, woody wetlands, and herbaceous wetlands) as well as a ratio of low to high developed land, calculated by taking the amount of open-to-low developed land and dividing by the amount of medium-to-high developed land.

We also collected parent-reported natural features near the child's home (and school or daycare if applicable). This questionnaire (adapted from Tilt, Unfried, \& Roca, 2007), asks about the presence of nine types of natural features within an approximate half mile distance from home or school. The total number of features near home (or the average of home and school/daycare if both included) was used to calculate parent-reported

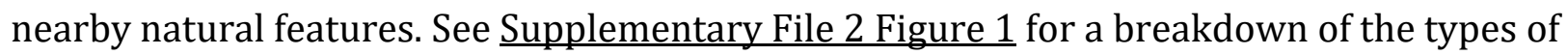
natural features near the homes and schools of the children in this sample. 
Additionally, the types of children's play environments outside of school and daycare hours and during school/daycare hours (if applicable) were assessed. The play environments questionnaire (adapted from Amoly et al., 2014; Faber Taylor \& Kuo, 2011) asked parents to indicate the environments that their child typically plays in most of the time during a warm week in autumn or spring. Play in more natural versus more built spaces was calculated by taking the number of natural play environments (big trees and grass, open grass, "wild" places, waterfronts, deserts, and farms) and subtracting the number of built or indoor environments (deep indoors, indoors with windows, paved or built places, public indoors). The average number of natural features, play environments, and other demographic data are presented separately by age year in Supplementary File 2 Table 1.

Lastly, we asked parents to fill out the Strengths \& Difficulties Questionnaire (Goodman, Lamping, \& Ploubidis, 2010), which assesses peer problems, conduct problems, emotional problems, hyperactivity/ inattentiveness, and prosocial behaviors.

Not all parents provided full questionnaire data during the study. Of the usable sample of 239 children, age and gender were collected for all 239 participants, 235 have basic demographic information (ethnicity, income, parental education), 195 provided their home zip code, 200 completed the parent-reported nearby natural features questionnaire, 171 completed the typical play environments questionnaire, and 151 completed the Strengths and Difficulties Questionnaire (see Supplementary File 2 Table 2 for full breakdown). 


\subsection{Data Analysis}

\subsubsection{Data Structure and Cleaning}

The output from the picture sorting task was 10 trials of pictures ranked from 1 (least preferred in trial) to 4 (most preferred in trial). Occasionally, participants accidentally hit the advance button twice in a row, skipping the trial. To account for this, trials that had a duration less than 1 second were removed from the data.

The average rating used for demonstration purposes and data visualization (in Figure 3 ) was calculated by taking the average position of each photo across the 4 trials in which it occurred, resulting in a value between 1 (always chosen as least preferred) and 4 (always chosen as most preferred) for each picture. For subsequent statistical analyses, these average ratings were sorted from highest to lowest to create a full 1 to 10 ranking of all images in the set. In the case where multiple images had the same average rating, the higher ranking was given to the image that was more preferred in the trial(s) that included both images. Because the statistical analysis procedures used (described in the Statistical Analysis section) are not easily graphed, these average ratings were used to visualize the pattern of results.

A subset of adult participants (15 out of 182) were excluded from analysis after being identified as likely completing the task in reverse. This assessment was based on having both exceptionally low ratings ( 1 to 1.75 out of 4 ) of the very high aesthetic value nature images and high ratings (3.25 to 4 ) of the very low aesthetic value urban images. The very high aesthetic value nature images and very low aesthetic value urban images 
received very reliable ratings in previous stimulus validation studies and across the rest of the adult sample in the current study. Given that the adults were given the instructions but did not do any practice trials with an experimenter, and on a few occasions parents realized they were doing the task in reverse and told the experimenter this was the case, we felt confident that these adults were likely not paying close attention to the frown/smile anchors and simply made their rankings backwards.

\subsubsection{Statistical Analysis of Task Data}

As the task data were ordinal and included repeated measures, we conducted regression analyses using a proportional odds mixed model (McCullagh, 1980), fit using the “ordinal” package (Christensen, 2018) in R 3.5.1 (R Foundation for Statistical Computing, www.rproject.org). This analysis models the effect of predictors across the "cut-points" between categories of the ordinal criterion variable. In the case of our data, there were 10 categories (ranks 1 through 10) resulting in nine cut-points. The proportional odds model predicts the log odds of a given response being below each cut-point, under the assumption that a predictor's effects do not significantly differ across cut-points (i.e., the proportional odds assumption). If the proportional odds assumption is met, the model yields cumulative odds ratios that do not depend on the specific cut-points used. To test the proportional odds assumption, we modelled predictor separately as nominal effect and as an ordinal effect, and the model fits were compared via likelihood-ratio test. In all cases, the model fits did not significantly differ, indicating that the proportional odds assumption was met (i.e., the effect of predictors did not differ across cut-points). Maximum likelihood parameter estimates were obtained using an adaptive Gauss-Hermite quadrature approximation using 
11 quadrature points (Lesaffre \& Spiessens, 2001), implemented through the nlminb function in R. Participant was included as a random intercept in our analyses to account for repeated measures. To specifically analyze age-related changes in the very high aesthetic value nature and very low aesthetic value urban categories, proportional odds modelling was conducted using the lrm function in the R package 'rms' (Harrell, 2018).

\subsubsection{Analysis of Environmental Exposure and $S D Q$ variables}

To examine the relation between individual differences in children's nature exposure and cognitive functioning as measured by the Strengths \& Difficulties Questionnaire, a multiple imputation procedure (Rubin, 1987) was first employed to handle participants with varying amounts of missing questionnaire data. Multiple imputation was performed using the "mice" package in R (van Buuren, 2018). Results with and without multiple imputation were very similar, and as such, the results reported in the manuscript are those from the raw (not imputed) data. Results including imputation to account for data missingness can be found in Supplementary File 2 .

\subsubsection{Analysis of Parent-Child Similarity of Preference.}

To analyze whether children's preferences reflect those of their parents, the Euclidean distance between children's rankings of images and their parents' rankings were calculated and compared to non-parents. For this analysis, the subset of data was used which only included independent parent-child pairs, as the inclusion of multiple siblings with a single parent would directly violate the assumption of independent samples in subsequent analyses. A standard distance calculation was performed, using the image ranks for all 10 images for kids and parents: 
GRADUAL DEVELOPMENT OF NATURE PREFERENCES

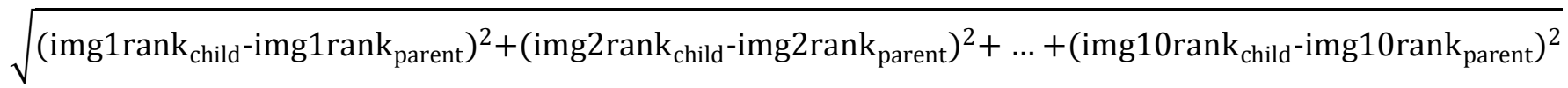

This calculation was also performed on each child and every adult other than the child's parent in the same picture set. These calculations were then averaged, to create a value of the average distance of the child and the $\mathbf{n}$ other adults. That is, (

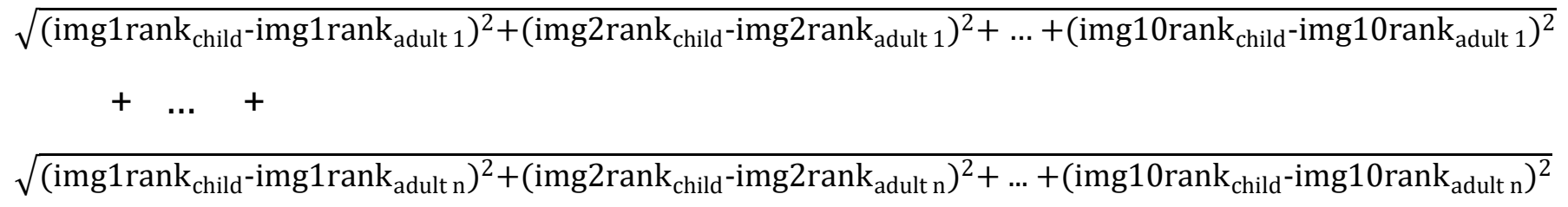

\subsubsection{Supplementary Analyses}

A series of additional analyses were conducted to promote transparency and to ensure the reliability of our results. These results are reported in Supplementary File 2, and any discrepancies between original results and these analyses are described in the results section. For analyses that involved the adult participants (section 3.1), we also conducted the same statistical tests including those 15 adult participants that were excluded from our sample. All analyses involving child participants were conducted using only the noncorrelated subset (described in 2.2 Participants) which excludes siblings. Any analyses that involved our multiple imputation procedure (section 3.3) are also reported in this supplement. 
GRADUAL DEVELOPMENT OF NATURE PREFERENCES

\section{Results}

\subsection{Environmental Preferences differ between Children and Adults}

To test for preference differences between adults (i.e., parents and guardians in the current study) and children, a proportional odds mixed model was conducted using rank (1-10) as an outcome variable, environment type (nature/urban) and aesthetic value (high/low) as within-subject predictors, categorical age (adult/child) as a betweensubjects predictor, and participant as a random variable. The model using the full interaction of predictor variables did not yield a significant 3-way interaction, and did not differ in goodness of fit from the model including only 2-way interactions, so the results of the more parsimonious model are reported.

A significant interaction of categorical age and environment type was found $(B=$ $1.44, Z=11.45, O R=0.27,95 \% \mathrm{CI}[0.18,0.30], p<0.001)$, where children showed a greater preference for urban images compared to adults. Adults and children also differed in their preference for images based on aesthetic value $(B=0.16, Z=2.90, O R=0.70,95 \% \mathrm{CI}[0.54$, 0.89 ], $p=0.003$ ). Specifically, adults preferred the high aesthetic value images (compared to low aesthetic value ones) to a greater extent than did children (see Figure 3). When the 15 adults previously excluded (due to probable reversed responding) were included in this analysis, the interaction between categorical age and aesthetic value was no longer significant (p.s. $=0.3$ ) but the interaction with environment was unaffected.

\subsection{Children's Environmental Preferences}

To test for children's preferences and how they may be influenced by age, a proportional odds mixed model was again employed using rank (1-10) as an outcome 
variable, environment type (nature/urban) and aesthetic value (high/low) as withinsubject factors, child age as a continuous predictor, and subject as a random variable.

\subsubsection{Group Effects}

Results of this analysis showed a main effect of environment, where children generally preferred the urban environments compared to natural ones $(B=-0.83, Z=-7.10$, $O R=0.44,95 \% \mathrm{CI}[0.35,0.55], p<0.001)$. There was also a main effect of aesthetic value where children exhibited greater preferences for high aesthetic value images $(B=1.73, Z=$ $14.5, O R=5.65,95 \% \mathrm{CI}[4.47,7.14], p<0.001)$. A significant interaction of environment and aesthetic value was also found $(B=0.70, Z=4.31, O R=0.50,95 \% \mathrm{CI}[0.36,0.68], p<$ 0.001) where children exhibited a larger ranking difference between high and low aesthetic value nature images compared to urban images (see Figure 3). (Group effect analyses for adults can be found in Supplementary File 2). 


\section{Figure 3. Preference for Environment Types in Adults and Children}

Plotted are the average ratings for each image type (VHA_N = Very High Aesthetic value Nature, HA_N = High Aesthetic value Nature, LA_N = Low Aesthetic value Nature, HA_U = High Aesthetic value Urban, LA_U = Low Aesthetic value Urban, VLA_U = Very Low Aesthetic value Urban) in the adult and child samples. The boxes represent the four conditions that are completely crossed for statistical analysis. In this chart, higher ratings (closer to 4) represent more favored image types and lower ratings (closer to 1) represent less liked image types. Error bars indicate +/- SEM.

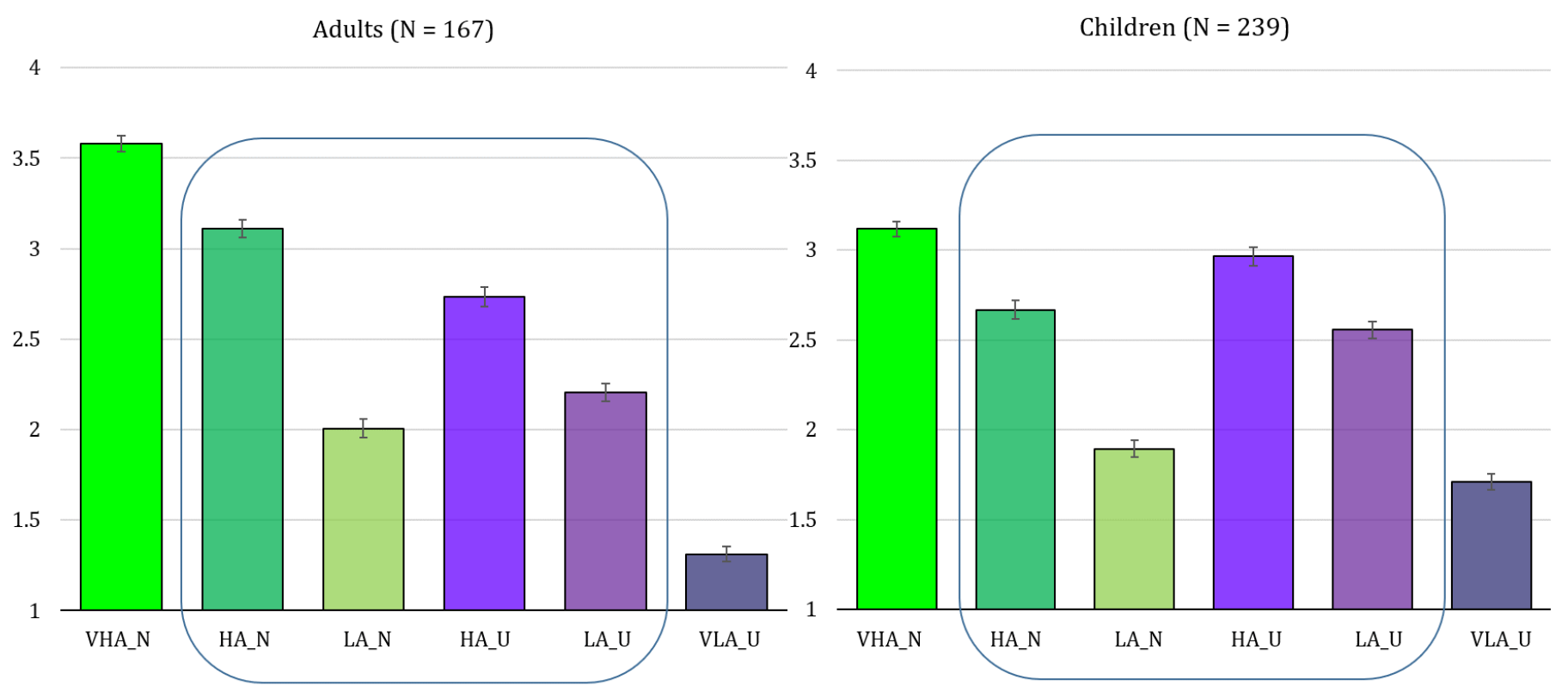

\subsubsection{Age-Dependent Preferences}

Importantly, both children's preference for environment type and aesthetic value showed significant interactions with age. With increasing age, children showed a lessened preference for urban environments over natural ones $(B=0.32, Z=4.30, O R=1.39,95 \% \mathrm{CI}$ $[1.18,1.63], p<0.001)$. Additionally, children preferred images of high over low aesthetic 
value to a greater degree with increasing age $(B=0.27, Z=3.31, O R=1.31,95 \% \mathrm{CI}[1.11$, 1.53], $p<0.001$; Figure 4).

Age related changes in the very high aesthetic value nature images and very low aesthetic value urban images were examined in separate proportional odds regressions, predicting the rank of the image (1 to 10) by child age. A significant effect of age was found on rankings of very high aesthetic value nature images $(B=-0.43, Z=-3.62, O R=0.65,95 \%$ CI $[0.51,0.82], p<0.001)$, where higher preference rankings were associated with increasing child age. There was also a significant effect of age on ranking of very low aesthetic value urban images $(B=0.68, Z=5.59, O R=1.98,95 \%$ CI $[1.56,2.52], p<0.001)$. For this category, as age increased, children's preferences for these images decreased (Figure 4). In summary, as children aged, their preferences began to look more similar to those of adults. 
Figure 4. Developmental Changes in Environmental and Aesthetic Value Preference

The top panels represent differences calculated from average ratings, plotted across age groups. The environment preference measure ("Nature - Urban") displayed in the upper left panel was calculated by subtracting the average of the high and low aesthetic value Urban categories from the average of the high and low aesthetic value Nature categories. Similarly, the aesthetic value measure ("High - Low Aesthetic Value") displayed in the upper right panel was calculated by subtracting the average of ratings for Low Aesthetic value Nature and Urban images from the High Aesthetic value Nature and Urban images. The bottom panels represent average ratings for the Very High Aesthetic value Nature category (lower left) and Very Low Aesthetic value Urban category (lower right), divided across age bins. Error bars represent + /- SEM.

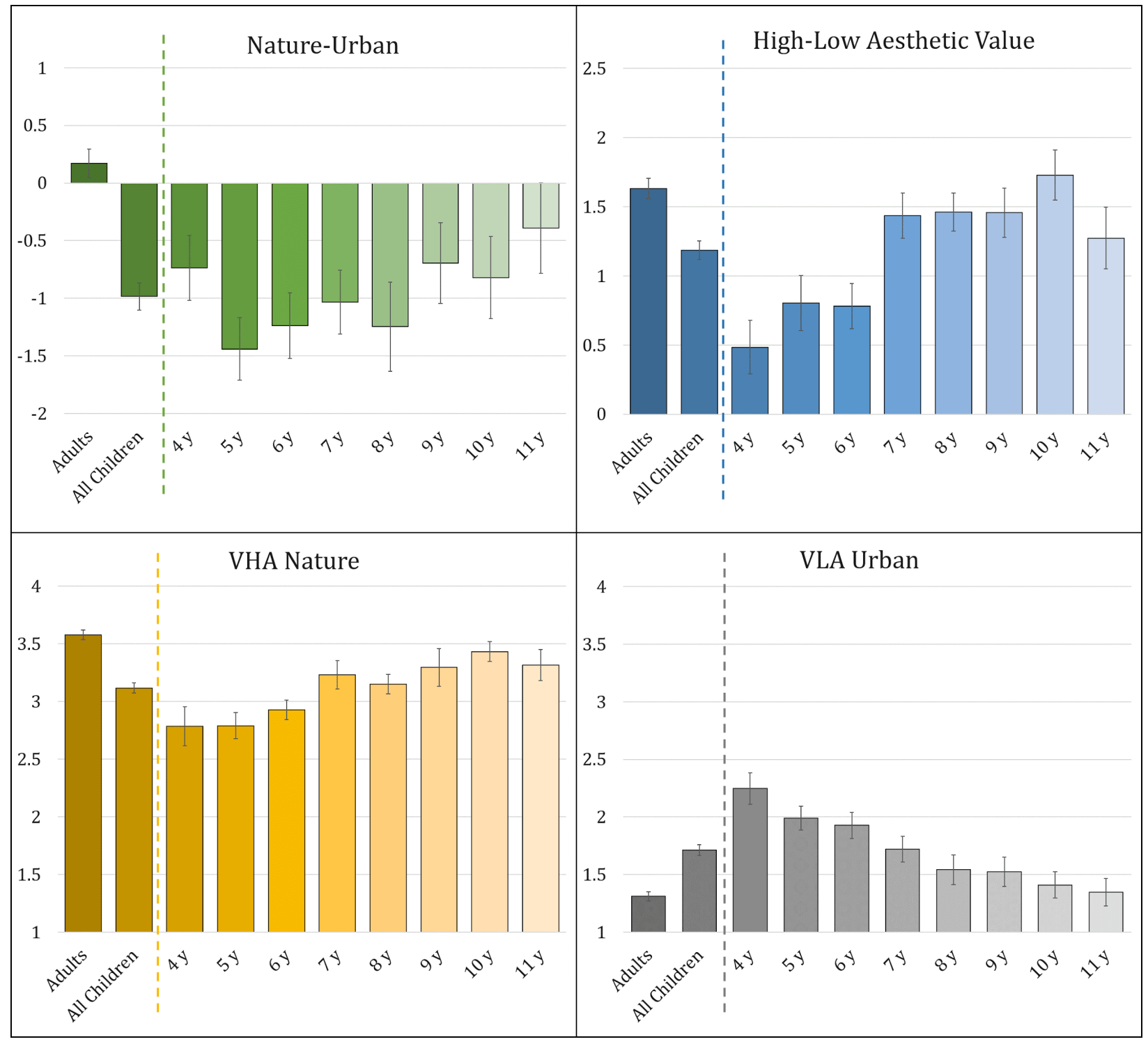




\subsection{Relations between nature exposure, nature preference, and children's behavior}

A goal of this study was to examine whether any of the observed benefits of nature exposure on children's cognitive, social, and emotional functioning could be explained by individual differences in children's nature preferences. Another aim was to see if nature exposure was linked to preference.

\subsubsection{Nearby Nature Predicts Children's Attention}

Based on extensive prior research that looked at the effects of nature exposure on children's attentional functioning, a confirmatory analysis was conducted examining the effects of nearby nature and play in nature on the parent-reported hyperactivity/ inattentiveness subscale from the SDQ. As predicted, greater parent-reported nearby natural features were correlated with lower scores on the SDQ hyperactivity/ inattentiveness $(r=-0.21,95 \% \mathrm{CI}[-0.36,-0.05], p=0.009)$. However, income was also negatively correlated with hyperactivity/ inattentiveness $(r=-0.21,95 \%$ CI $[-0.36,-0.04], p$ $=0.015)$. Thus, separate analyses were run to see whether natural features were still predictive when income was included in the model. Results from a linear regression indicated that both greater parent-reported natural features and higher income were significant independent predictors of children's inattentiveness/hyperactivity (Natural features: $B=-0.31, p=0.015 ; \eta^{2}=0.04$; Income: $\left.B=-0.31, p=0.023, \eta p^{2}=0.04\right)$. When this analysis was conducted on the non-correlated subset of the data which reduced our sample size for this analysis, this effect became non-significant $(p=0.1)$. Surprisingly, play in more natural environments was not significantly related to this SDQ measure $(p s=0.2)$. 


\subsubsection{Other Nature-Behavior Relations}

An exploratory analysis was conducted to examine whether parent-reported interactions with nearby nature (natural features near home/school), parent-reported play in natural over built environments, or objective measures of nearby nature (zip code based NLCD measures of natural features and high versus low developed space) were correlated with any of the other SDQ subscales: conduct problems, emotional problems, peer problems, and prosociality.

Results of the full correlation matrix can be found in Table 4. As expected, there were strong correlations between NLCD natural features and parent-reported nearby natural features $(r=0.44,95 \% \mathrm{CI}[0.32,0.55], p<0.001)$, as well as NLCD natural features and play in natural environments $(r=0.32,95 \% \mathrm{CI}[0.18,0.44], p<0.001)$.

More parent-reported nearby natural features were modestly correlated with lower scores on the SDQ conduct problems subscale $(r=-0.15,95 \%$ CI $[-0.3,0.01], p=0.077)$, but was not related to any other SDQ subscales. Play in natural environments and nearby natural features calculated from zip-code based national land cover data (NLCD) were not related to any SDQ measures (all $p>0.13$ ). 
Table 4. Correlation matrix of nearby nature, SDQ, demographics, and child environmental preference

Correlation coefficients are listed for all correlations between parent-reported and objective nature, SDQ subscales, SES measures, and children's environmental preferences. Coefficients highlighted in blue with ${ }^{* *}$ indicate a $p$-value of less than 0.01 , and those highlighted in green with * indicate $p<0.05$.

$\begin{array}{llllllllll}\text { PR Nat } & \text { NLCD } & \text { NLCD } & \text { SDQ } & \text { SDQ } & \text { SDQ } & \text { SDQ } & \text { SDQ } & \text { Income Mom } & \text { Child } \\ \text { Play } & \text { Nat Feat } & \text { Dev } & \text { Emot } & \text { Cond } & \text { Inatt/ } & \text { Peer } & \text { Prosoc } & \text { Ed } & \text { Env Pref } \\ & & & & & \text { Hyper } & & & & \end{array}$

\begin{tabular}{|c|c|c|c|c|c|c|c|c|c|c|c|}
\hline $\begin{array}{l}\text { PR Nat } \\
\text { Feat }\end{array}$ & $.37 * *$ & $.44^{* *}$ & $.32^{* *}$ & .00 & -.15 & $-.21^{* *}$ & -.04 & .15 & .14 & .01 & -.02 \\
\hline $\begin{array}{l}\text { PR Nat } \\
\text { Play }\end{array}$ & & $.32 * *$ & $.19 * *$ & -.07 & -.09 & -.11 & -.08 & .13 & .02 & .14 & .00 \\
\hline $\begin{array}{l}\text { NLCD Nat } \\
\text { Feat }\end{array}$ & & & $.56^{* *}$ & -.13 & -.09 & -.12 & -.1 & .14 & -.06 & .01 & -.07 \\
\hline NLCD Dev & & & & -.08 & -.08 & -.1 & .02 & .14 & .05 & .06 & -.01 \\
\hline SDQ Emot & & & & & $.31^{* *}$ & $.36 * *$ & $.34^{* *}$ & $.26^{* *}$ & $-.24^{* *}$ & -.04 & .00 \\
\hline SDQ Cond & & & & & & $.49 * *$ & .08 & $-.43 * *$ & -.14 & -.09 & -.13 \\
\hline SDQ Inatt & & & & & & & .16 & $-.34 * *$ & $-.21 *$ & $-.19 *$ & -.05 \\
\hline SDQ Peer & & & & & & & & -.12 & -.15 & -.02 & .05 \\
\hline SDQ Pros & & & & & & & & & .05 & .05 & -.03 \\
\hline Income & & & & & & & & & & $.45^{* *}$ & -.13 \\
\hline Mom Ed & & & & & & & & & & & -.08 \\
\hline
\end{tabular}

[Legend: PR Nat Feat $=$ Parent-reported nearby natural features, PR Nat Play $=$ Parent-reported measure of child's play in nature over built space, NLCD Nat Feat = natural features calculated from zip-code level NCLD data, NLCD Dev = ratio of open/low to medium/high developed space from zipcode level NLCD data, SDQ Emot = Emotional problems subscale, SDQ Conduct = Conduct problems subscale, SDQ Inatt = Inattentive $/$ Hyperactive subscale, SDQ Peer = Peer problems subscale, SDQ Pros $=$ Prosocial behavior subscale, Income $=$ family household income, Mom Ed = Maternal Education, Child Env Pref = Child's preference for natural over urban environments] 


\subsection{Children's Nature Preferences and Environmental Exposure}

To examine whether individual differences in nature preferences related to their nearby nature exposure, a metric of nature versus urban preference was also examined in the correlations. This metric was calculated by taking the average rank for the four nature images and subtracting the average rank of the four urban images.

\subsubsection{Preference and Exposure Across All Children}

The results of these correlations indicated no significant correlations between children's environmental preferences and any of the nearby nature exposure or behavioral measures (see Table 4).

\subsubsection{Interaction of Nature Preference, Environmental Exposure, and Age}

A proportional odds mixed model was conducted to examine the relationship between nearby natural features, preference for natural environments, and age. Results of this analysis showed a significant 3-way interaction of child age, environmental preference, and parent-reported nearby natural features $(B=0.39, Z=4.6, O R=1.49,95 \%$ CI $[1.26$, 1.76], $p<0.001$ ). To unpack this interaction, follow up proportional odds mixed models were performed separately on each of the datasets include 4-5 year-olds, 6-7 year-olds, 8-9 year-olds, and 10-11 year-olds. None of the interactions between nearby natural features and preferences reached significance in the 4-to-5, 6-to-7, or 8-to-9-year old children. However, in the 10-to-11-year old group, this interaction was significant $(B=0.68, Z=3.45$, $O R=1.96,95 \% \mathrm{CI}=[1.33,2.89], p<0.001)$. The results of this analysis suggested that with the older children, nearby natural features were positively related to a greater preference for natural over urban images, but this was not the case for children under 10. 


\subsection{Parental Influences on Children's Preferences}

Results of a repeated measures ANOVA comparing child-parent distance and childnonparent average distance indicated that the distance between a child and their parent $(M$ $=9.96, S D=3.17)$ was smaller than that between the child and the average non-parent $(M=$ 13.8, $\left.S D=1.0 ; F(1,143)=152.9, p<0.001, \eta^{2}=0.4\right)$. This shorter distance indicates that, overall, children demonstrated more similar preferences to those of their own parent relative to parents of other children viewing the same images.

To see whether parent-child similarity was affected by child age, a mixed model ANOVA was run predicting distance from child-parent pairs versus child-non-parent pairs and child year of age, with a random effect for subject. This analysis yielded a significant interaction of age and parent vs. non-parent similarity $\left(F(7,136)=2.83, p=0.009, \eta^{2}=0.08\right.$; Figure 5), where children's preferences were more similar to those of their parent over other adults with increasing age. 


\section{Figure 5: Age effects for Parent-Child Preference Similarity}

Boxplot depicting the distance between preferences of children and their parents (purple) and children with the average parent rating the same picture set (green) plotted separately by year of age.

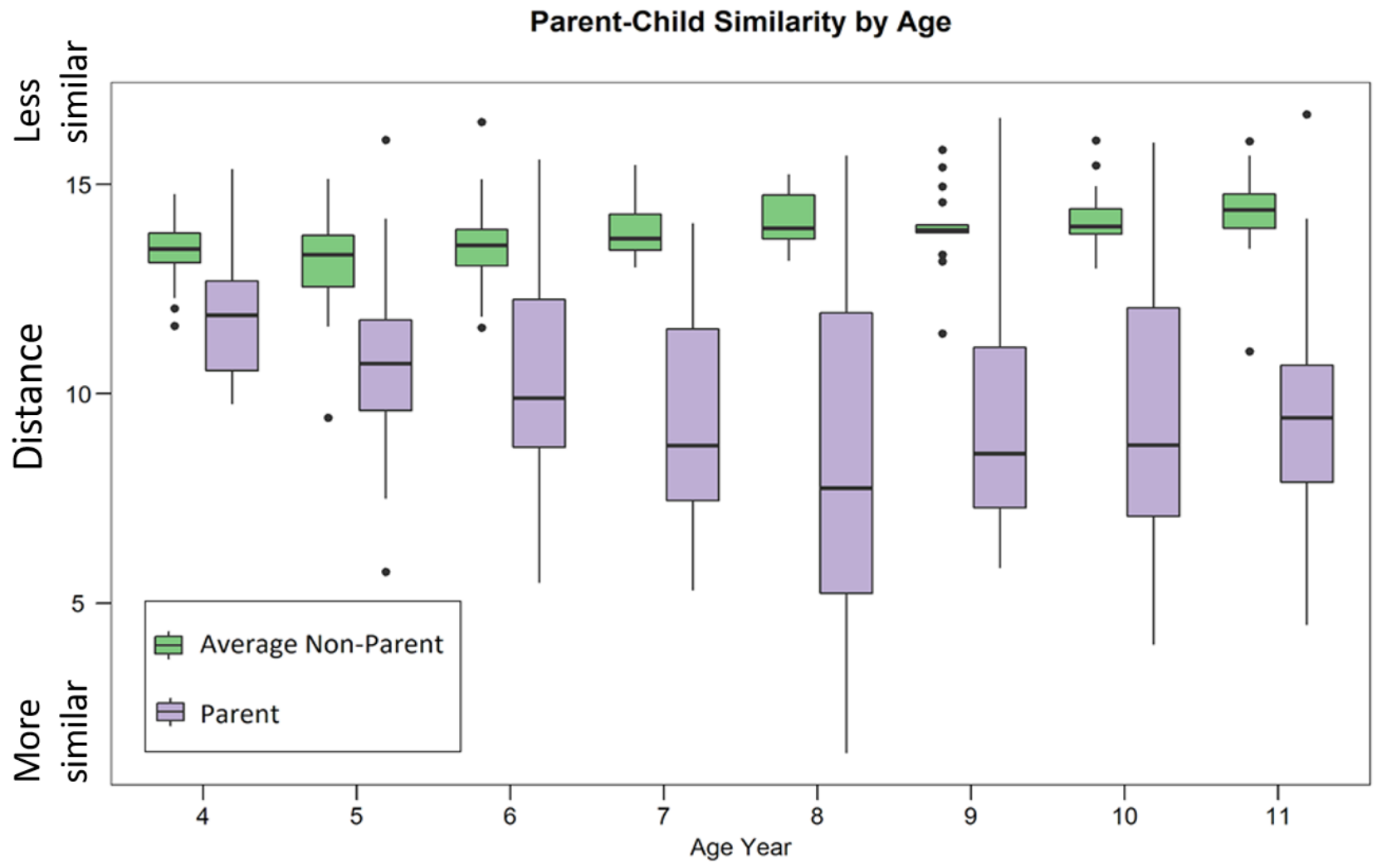

\section{Discussion}

Whether the pervasive preferences that adults show for nature (Kaplan \& Kaplan, 1989) also exist in young children is an important, unanswered question. This study attempted to address this outstanding issue, and to understand how and when such preferences develop. Unexpectedly, this study found that children (4 to 11 years) have robust urban preferences, counter to adult preferences. However, this preference for urban environments decreased, almost linearly, with children's age. This key finding (among 
others) from the current study provide greater support for a learned affinity for nature, rather than an affinity that has been genetically programmed and present from birth. If an innate, biophilic response is indeed responsible for the preference for nature observed in adults, this developmental trajectory fits most readily with a biologically prepared learning account. This account has also been previously proposed in cross-cultural studies of rural vs. urban environmental effects on attention over development (Kardan et al. 2017).

Young children in our study exhibited a considerable urban preference, but this counterintuitive finding does not appear to have an obvious cause. One feature that strongly relates to children's preferences is stimulus novelty (Cantor \& Cantor, 1964). A novelty account would make the argument that children find the urban images more interesting because the scenes are less familiar. This is an unlikely explanation for our results as overall, parent-reported and objective measures of nature near children's homes or schools were not related to their preferences (i.e. a more urban home environment did not predict preference for nature and vice versa).

The current study also found that children's preferences looked more similar to their parent's with increasing age, which suggests that with more time with one's parents/caregivers, child and parent preferences converge as compared to more divergence at younger child ages. Note, this is not just due to older kids exhibiting preferences more like adults in general, as these effects were specific to similarities in preference with one's own parents/caregivers vs. the other adults in the study (i.e., a difference score). Thus, it appears that parental preferences may be a key influence on what children prefer, but this influence seems to manifest later in development. This study has identified that environmental factors such as nearby natural features and parental 
preferences affect older child environmental preferences more than younger child environmental preferences, but our data cannot speak to any additional mechanisms or identify what specific factors might impact the preferences of young children. The observed effects do, however, provide an exciting avenue for additional research to identify what underlying mechanisms drive these preferences.

This study also provides support for the idea that children need not prefer natural environments to reap the cognitive benefits from interacting with natural environments. We identified a significant correlation between having greater nearby nature in children's home and school environments and reduced child inattentiveness and hyperactivity, but these effects were not related to children's preferences. This pattern is consistent with Attention Restoration Theory (Kaplan \& Berman, 2010), which suggests that nature exposure improves cognitive functioning through replenished attentional capacity, and not through preference-driven mood changes. Additionally, if young children do not generally prefer nature, then it is possible that the cognitive benefits observed for children after nature exposure from other studies may also not be due to preference (Dadvand et al., 2015; Faber Taylor \& Kuo, 2011; Faber Taylor \& Kuo, 2009; Wells, 2000). However, as this study does not involve directly manipulating nature exposure for these children, these data do not lend themselves to strong conclusions on the role of preference on cognitive effects. To directly test this, future empirical studies that utilize nature interventions on children's cognitive functioning should consider including a measure of children's environmental preferences, but that is careful to use stimuli that decouple preference from environment type. 
Like all studies, our study is not without limitations. First, though we are using the same anchors and verbal descriptions of preference in both adults and children (i.e., "like", "favorite"), it is impossible to tell with the current design whether we are tapping into the same psychological construct. For example, it could be that children and adults differentially weight the desire to be in a given environment when making a preference evaluation. Again, future research would be required to rule out this explanation. Another limitation relates to the age range chosen. Though a pilot study indicated that we would have difficulty collecting data from children under 4 years of age with this paradigm, there are potentially fascinating and important developmental effects in infants and younger children that require investigation with age-appropriate experimental procedures. Our 11year old participants did not display preference patterns identical to those of adults, and as such, extending this work into older ages would be required to fully examine the complete developmental trajectory. This is particularly important as previous research has shown that environmental preferences during the adolescent years may be quite different from those displayed in adulthood (Balling \& Falk, 1982; Kaplan \& Kaplan, 2002). Lastly, though the effect we observed relating attentional problems to less nearby nature has been found in other studies (Faber Taylor \& Kuo, 2009; Faber Taylor \& Kuo, 2011; Dadvand et al., 2015; Amoly et al., 2014; Kuo, Barnes, \& Jordan, 2019), it is still correlational and uses subjective, parent reported inattentiveness, which is a limitation of this survey-based data. Importantly, our study decoupled image preference from environment type and used multiple picture sets to allow for generalizing above a particular set of images. It would be beneficial to implement this paradigm with a wider array of images to ensure that these results can be generalized further, though it may be difficult to increase the image 
corpus much, as young children only have the attention span to perform the task for a few minutes. It was therefore outside the scope of the current study to include additional image sets that may not be preference-equated but chosen to represent more variety in nature (i.e. lakes, forests, deserts) or urban (i.e. houses, cityscapes) scenes. However, future research on this topic which includes a wider array of image types would be a valuable addition to the current results.

In summary, these data provide the first step in a broader series of studies that can attempt to understand why children and adults differ in their environmental preferences, what drives age-dependent changes in preference, and whether nature preferences are necessary for all, some, or none of the observed cognitive, emotional and health benefits of nature exposure in children. 
Acknowledgements:

We are grateful to Jillian Bowman, Anjali Gundeti, Tanvi Lakhtakia, Anabella Pinton, Claire Sampson, Jason Steinberg, and Lena Woo for their assistance in data collection. We also thank Michael Ingram for his help coding the task. We would like to dedicate this article to Dr. Stephen Kaplan (1936-2018), who was a great mentor, friend and advisor to M.G.B. and was an inspiration to much of this work.

\section{Funding:}

This work was supported in part by grants from the TKF Foundation, the John Templeton Foundation (University of Chicago Center for Practical Wisdom and the Virtue, Happiness, and Meaning of Life Scholars Group), and the National Science Foundation [Grant BCS1632445]. Partial support comes from the international research fellowship grant from the Swedish Research Council [reference no 2015-00190]. 
GRADUAL DEVELOPMENT OF NATURE PREFERENCES

References:

Amoly, E., Dadvand, P., Forns, J., López-Vicente, M., Basagaña, X., Julvez, J., ... Sunyer, J. (2014). Green and blue spaces and behavioral development in Barcelona schoolchildren: the BREATHE project. Environmental Health Perspectives, 122(12), $1351-1358$.

Balling, J. D., \& Falk, J. H. (1982). Development of Visual Preference for Natural Environments. Environment and Behavior, 14(1), 5-28.

Berman, M. G., Hout, M. C., Kardan, O., Hunter, M. R., Yourganov, G., Henderson, J. M., ... Jonides, J. (2014). The perception of naturalness correlates with low-level visual features of environmental scenes. PloS One, 9(12), e114572.

Berman, M. G., Jonides, J., \& Kaplan, S. (2008). The Cognitive Benefits of Interacting With Nature. Psychological Science, 19(12). Retrieved from http://journals.sagepub.com/doi/pdf/10.1111/j.1467-9280.2008.02225.x

Berman, M. G., Kross, E., Krpan, K. M., Askren, M. K., Burson, A., Deldin, P. J., ... Jonides, J. (2012). Interacting with nature improves cognition and affect for individuals with depression. Journal of Affective Disorders, 140(3), 300-305.

Berto, R. (2005). Exposure to restorative environments helps restore attentional capacity. Journal of Environmental Psychology, 25(3), 249-259.

Bratman, G. N., Hamilton, J. P., Hahn, K. S., Daily, G. C., \& Gross, J. J. (2015). Nature experience reduces rumination and subgenual prefrontal cortex activation. Proceedings of the National Academy of Sciences of the United States of America, 112(28), 8567-8572. 
Cantor, J. H., \& Cantor, G. N. (1964). Observing behavior in children as a function of stimulus novelty. Child Development, 35, 119-128.

Christensen, R. H. B. (2018). Package "ordinal."

Coburn, A., Kardan, O., Kotabe, H., Steinberg, J., Hout, M. C., Robbins, A., ... Berman, M. G. (2019). Psychological responses to natural patterns in architecture. Journal of Environmental Psychology, 62, 133-145.

Corraliza, J. A., Collado, S., \& Bethelmy, L. (2012). Nature as a Moderator of Stress in Urban Children. Procedia - Social and Behavioral Sciences, 38, 253-263.

Crompton, J. L. (2001). The Impact of Parks on Property Values: A Review of the Empirical Evidence. Journal of Leisure Research, 33(1), 1-31.

Dadvand, P., Nieuwenhuijsen, M. J., Esnaola, M., Forns, J., Basagaña, X., Alvarez-Pedrerol, M., ... Sunyer, J. (2015). Green spaces and cognitive development in primary schoolchildren. Proceedings of the National Academy of Sciences of the United States of America, 112(26), 7937-7942.

Faber Taylor, A., \& Kuo, F. E. (2009). Children with attention deficits concentrate better after walk in the park. Journal of Attention Disorders, 12(5), 402-409.

Faber Taylor, A., \& Kuo, F. E. M. (2011). Could Exposure to Everyday Green Spaces Help Treat ADHD? Evidence from Children's Play Settings. Applied Psychology. Health and Well-Being, 3(3), 281-303.

Fleischer, A. (2012). A room with a view—A valuation of the Mediterranean Sea view. Tourism Management, 33(3), 598-602.

Goodman, A., Lamping, D. L., \& Ploubidis, G. B. (2010). When to use broader internalising and externalising subscales instead of the hypothesised five subscales on the Strengths 
GRADUAL DEVELOPMENT OF NATURE PREFERENCES

and Difficulties Questionnaire (SDQ): data from British parents, teachers and children. Journal of Abnormal Child Psychology, 38(8), 1179-1191.

Harrell, F. E., Jr. (2018). Package "rms."

Hartig, T., Evans, G. W., Jamner, L. D., Davis, D. S., \& Garling, T. (2003). Tracking restoration in natural and urban field settings. Journal of Environmental Psychology, 23, 109-123.

Ibarra, F. F., Kardan, O., Hunter, M. R., Kotabe, H. P., Meyer, F. A. C., \& Berman, M. G. (2017). Image Feature Types and Their Predictions of Aesthetic Preference and Naturalness. Frontiers in Psychology, 8, 632.

Joye, Y., \& De Block, A. (2011). "Nature and I are Two": A Critical Examination of the Biophilia Hypothesis. Environmental Values, 20(2), 189-215.

Joye, Y., Steg, L., Ünal, A. B., \& Pals, R. (2016). When complex is easy on the mind: Internal repetition of visual information in complex objects is a source of perceptual fluency. Journal of Experimental Psychology. Human Perception and Performance, 42(1), 103114.

Joye, Y., \& van den Berg, A. (2011). Is love for green in our genes? A critical analysis of evolutionary assumptions in restorative environments research. Urban Forestry \& Urban Greening, 10(4), 261-268.

Kahn, P. H., Jr. (1997). Developmental psychology and the biophilia hypothesis: Children's affiliation with nature. Developmental Review: $D R, 17(1), 1-61$.

Kaplan, R., \& Kaplan, S. (1989). The Experience of Nature: A Psychological Perspective. New York: Cambridge University Press. 
Kaplan, R., \& Kaplan, S. (2002) Adolescents and the natural environment: A time out? In P. H. Kahn, Jr. \& S. R. Kellert (Eds.) Children and nature: Psychological, sociocultural, and evolutionary investigations (Pp. 227-258). Cambridge, MA: MIT.

Kaplan, R., \& Yang, B. (1990). The perception of landscape style: a cross-cultural comparison. Landscape and Urban Planning, 19, 252-261.

Kaplan, S. (1995). The restorative benefits of nature: Toward an integrative framework. Journal of Environmental Psychology, 15(3), 169-182.

Kaplan, S., \& Berman, M. G. (2010). Directed Attention as a Common Resource for Executive Functioning and Self-Regulation. Perspectives on Psychological Science: A Journal of the Association for Psychological Science, 5(1), 43-57.

Kaplan, S., Kaplan, R., \& Wendt, J. S. (1972). Rated preference and complexity for natural and urban visual material. Perception \& Psychophysics, 12(4), 354-356.

Kardan, O., Demiralp, E., Hout, M. C., Hunter, M. R., Karimi, H., Hanayik, T., Yourganov, G., Jonides, J., \& Berman, M. G. (2015a). Is the Preference of Natural versus Man-Made Scenes Driven by Bottom-up Processing of the Visual Features of Nature? Frontiers in Psychology 6 (April). https://doi.org/10.3389/fpsyg.2015.00471.

Kardan, O., Gozdyra, P., Misic, B., Moola, F., Palmer, L. J., Paus, T., \& Berman, M. G. (2015b). Neighborhood greenspace and health in a large urban center. Scientific Reports, 5, 11610.

Kardan, O., Shneidman, L., Krogh-Jespersen, S., Gaskins, S., Berman, M. G., \& Woodward, A. (2017). Cultural and Developmental Influences on Overt Visual Attention to Videos. Scientific Reports, 7(1), 11264. 
Kellert, S. R. (1993). The biological basis for human values of nature. The Biophilia Hypothesis, 42-69.

Kellert, S. R., \& Wilson, E. O. (1995). The Biophilia Hypothesis. Island Press.

Kotabe, H. P., Kardan, O., \& Berman, M. G. (2017). The nature-disorder paradox: A perceptual study on how nature is disorderly yet aesthetically preferred. Journal of Experimental Psychology. General, 146(8), 1126-1142.

Kuo, M., Barnes, M., \& Jordan, C. (2019). Do Experiences With Nature Promote Learning? Converging Evidence of a Cause-and-Effect Relationship. Frontiers in Psychology, 10, 305.

Lesaffre, E., \& Spiessens, B. (2001). On the effect of the number of quadrature points in a logistic random effects model: an example. Applied Statistics, 50(3), 325-335.

Mårtensson, F., Boldemann, C., Söderström, M., Blennow, M., Englund, J.-E., \& Grahn, P. (2009). Outdoor environmental assessment of attention promoting settings for preschool children. Health \& Place, 15(4), 1149-1157.

McCullagh, P. (1980). Regression Models for Ordinal Data. Journal of the Royal Statistical Society. Series B, Statistical Methodology, 42(2), 109-142.

Moore, R. C. (1986). The power of nature: Orientations of girls and boys toward biotic and abiotic play settings on a reconstructed schoolyard. Children's Environments Quarterly, $3(3), 52-69$.

Nielsen, T. S., \& Hansen, K. B. (2007). Do green areas affect health? Results from a Danish survey on the use of green areas and health indicators. Health \& Place, 13(4), 839-850.

Rubin, D. B. (1987). Multiple Imputation for Nonresponse in Surveys. 
Schertz, K.E. \& Berman, M.G. (2019). Understanding Nature and its Cognitive Benefits. Current Directions in Psychological Science. https://doi.org/10.1177/0963721419854100

Staats, H., Kieviet, A., \& Hartig, T. (2003). Where to recover from attentional fatigue: An expectancy-value analysis of environmental preference. Journal of Environmental Psychology, 23(2), 147-157.

Stenfors, C., Van Hedger, S., Schertz, K., Meyer, F., Smith, K., Norman, G., ... Berman, M. G. (2019). Positive Effects of Nature on Cognitive Performance across Multiple Experiments: Test Order but Not Affect Modulates the Cognitive Effects. Frontiers in Psychology 10: 1413.

Tilt, J. H., Unfried, T. M., \& Roca, B. (2007). Using objective and subjective measures of neighborhood greenness and accessible destinations for understanding walking trips and BMI in Seattle, Washington. American Journal of Health Promotion: AJHP, 21(4 Suppl), 371-379.

Ulrich, R. S. (1993). Biophilia, biophobia, and natural landscapes. The Biophilia Hypothesis, 7, 73-137.

Ulrich, R. S., Simons, R. F., Losito, B. D., Fiorito, E., Miles, M. A., \& Zelson, M. (1991). Stress recovery during exposure to natural and urban environments. Journal of Environmental Psychology, 11(3), 201-230.

van den Berg, A. E., Hartig, T., \& Staats, H. (2007). Preference for Nature in Urbanized Societies: Stress, Restoration, and the Pursuit of Sustainability. The Journal of Social Issues, 63(1), 79-96. 
Van Hedger, S. C., Nusbaum, H. C., Clohisy, L., Jaeggi, S. M., Buschkuehl, M., \& Berman, M. G. (2018). Of cricket chirps and car horns: The effect of nature sounds on cognitive performance. Psychonomic Bulletin \& Review. https://doi.org/10.3758/s13423-018$1539-1$

Van Hedger, S. C., Nusbaum, H. C., Heald, S. L. M., Huang, A., Kotabe, H. P., \& Berman, M. G. (2019). The Aesthetic Preference for Nature Sounds Depends on Sound Object Recognition. Cognitive Science, 43(5), e12734.

van Buuren, S. (2018). Package "mice."

Wells, N. M. (2000). At home with nature: Effects of "greenness" on children's cognitive functioning. Environment and Behavior, 32(6), 775-795.

Wells, N. M., \& Evans, G. W. (2003). Nearby Nature: A Buffer of Life Stress among Rural Children. Environment and Behavior, 35(3), 311-330. 